\title{
Clinical predictors of time to return to competition following hamstring injuries
}

\author{
Yannick Guillodo ${ }^{1}$ \\ Caroline Here-Dorignac ${ }^{1}$ \\ Bertrand Thoribé 2 \\ Gwénaelle Madouas ${ }^{3}$ \\ Marc Dauty 4 \\ Francois Tassery 5 \\ Alain Saraux ${ }^{1}$ \\ 1 Rhumatologie CHU Brest, France \\ 2 Clinique du sport Bordeaux Merignac, France \\ 3 Cabinet de médecine du sport du Questel, Brest, \\ France \\ ${ }^{4}$ Médecine physique et réadaptation, $\mathrm{CHU}$ Nantes, \\ France \\ ${ }^{5}$ Cabinet de médecine du sport, Le Havre, France
}

Corresponding author:

Yannick Guillodo

Rhumatologie $\mathrm{CHU}$ Brest, France

E-mail: docteur.guillodo@gmail.com

\section{Summary}

Objectives: hamstring strain injuries are the most common sports-related muscle injuries and one of the main causes of missed sporting events. Hypothesis: clinical findings reflecting hamstring injury severity at presentation predict time to sports resumption.

Design: cohort study (prognosis); Level of evidence, 2.

Methods: five sports medicine specialists at four sports medicine centers prospectively evaluated 120 athletes within 5 days of acute hamstring injury. Patients were interviewed and asked to evaluate their worst pain on a visual analog scale (VAS). Four physical criteria were assessed at baseline: bruising, tenderness to palpation, pain upon isometric contraction, and pain upon passive straightening. The same standardized rehabilitation protocol was used in all patients. A standardized telephone interview was conducted 45 days after the injury to determine the time tofull recovery ( $\leq 40$ days or $>\mathbf{4 0}$ days).

Results: by univariate analysis, clinical criteria associated with a full recovery time $>\mathbf{4 0}$ days were VAS pain score greater than 6 , popping sound injury, pain during everyday activities for more than 3 days, bruising, and greater than $15^{\circ}$ motion-range limitation. By multivariate analysis, only VAS pain score and pain during everyday activities were significantly associated with time to recovery $>\mathbf{4 0}$ days ( $53 \%$ sensitivity, $95 \%$ specificity).

Conclusion: the initial examination provides valuable information that can be used to predict the time to full recovery after acute hamstring injuries in athletes.

KEY WORDS: hamstring injuries, muscle, sport medicine, recovery of function.

\section{Introduction}

Sporting activities are the leading cause of muscles injuries, which account for a large proportion of all injuries. For example, in professional soccer players, muscle injuries contributed $31 \%$ of all injuries and $27 \%$ of total injury-related absences from play ${ }^{1}$. The diagnosis is usually based on the occurrence of sudden pain during a sporting activity, muscle tenderness to palpation, and pain upon resisted muscle contraction ${ }^{2}$. As with other sports injuries, evaluating severity is an important objective of the initial evaluation. Severity determines the time to sports resumption $^{3}$. Premature sports resumption may cause a recurrence. In a preliminary study ${ }^{4,5}$ of patients with sports-related injuries at various sites, we identified four baseline criteria predicting a time to sports resumption longer than 40 days: visual analog scale (VAS) pain score greater than $6 / 10$, pain during everyday activities for more than 3 days, tenderness to palpation, and greater than $15^{\circ}$ motion-range limitation compared to the uninjured side. However, this study was conducted in patients with a variety of sports-related injuries. Here, we studied an entirely new cohort of patients with hamstring injuries.

Hamstring injury is the most common musculoskeletal disorder and is prevalent in many sports, most notably those requiring maximal sprint accelerations $s^{1,2,6-8}$. Moreover, the treatment of hamstring injuries and games missed because of hamstring injuries result in substantial costs. A recent report (2010 injury report Australian Football League; http://mm.afl.com.au/portals/0/2010/aflinjuryreport2010_final.pdf) showed that hamstring injuries were the most prevalent sports-related lesions, with 20.6 missed games per club. Accurately predicting the time off competition is an important objective in athletes with hamstring injuries. 
Many studies identified clinical predictors of the time off competition after hamstring injuries $2,3,9-13$. The aim of this study was to evaluate whether clinical findings at baseline accurately predicted the time to recovery of the pre-injury level of performance in patients with hamstring injuries sustained during sporting activities.

\section{Methods}

Study population: between July 1, 2008, and April 1, 2009, five sports medicine specialists (YG, BT, GM, $\mathrm{MD}, \mathrm{FT}$ ) at four sports medicine centers in France included consecutive patients presenting within 5 days of sudden-onset posterior thigh pain during a sporting activity, with an inability to continue the training session or game. A history of injury to the same muscle group within the last 12 months was an exclusion criterion. Patients with a history of direct hamstring trauma were excluded.

Data collection: the following were recorded: demographic data; sporting activity; whether the pain occurred during a training session or a competition; previous muscle injuries; VAS fitness score; worst injuryrelated VAS pain score; time-course of the pain; whether the pain occurred during running, jumping, or kicking; site of the pain; whether a popping sound was heard at the time of injury; previous pain in the same muscle during the last week (pre-injury symptom); and number of days with muscle pain during everyday activities after the injury ${ }^{4,5}$.

All patients were examined by sports medicine specialists (10 years or more of experience with sports medicine), who performed a standardized physical examination within 5 days of injury onset. Four criteria were assessed during the physical examination: bruising; tenderness to palpation, with determination of the highest site of pain to palpation and measurement of the distance between this site and the palpated ischialtuberosity ${ }^{14}$; pain upon isometric contraction; and pain upon passive straightening of the limb, with measurement of motion-range limitation compared to the other limb, in degrees.

Follow-up modalities and the use of ultrasonography and/or magnetic resonance imaging (MRI) were at the discretion of the sports medicine specialists.

Rehabilitation program: the same written standardized rehabilitation program was used in all patients. This program consisted of physiotherapy, stretching, and strengthening exercises with progression from static to concentric to eccentric exercise, followed by training with progression from cycling to jogging to sprinting.

Follow-up: patients were interviewed on the phone 45 days after the initial injury by the sports medicine specialist who had performed the baseline clinical examination, using a standardized questionnaire to collect information on pain, rehabilitation, and progress toward sport resumption from mild exercise to the pre-injury level of activity and performance. Patients were divided into two outcome groups based on whether their time off competition was equal to or shorter than 40 days (early recovery) or longer than 40 days (late recovery) $)^{4,5}$.
Statistical analysis: the data were entered into a database then analyzed using the Statistical Package for the Social Sciences (SPSS 15.0, Chicago, IL). Associations between variables collected at baseline and time-to-recovery group were assessed by univariate analysis using the chi-square test (or Fisher's exact test where appropriate) and the MannWhitney test. Variables yielding $P$ values smaller than 0.20 were entered in a multivariate regression model with forward selection. Variables yielding $P$ values smaller than 0.05 in this model were considered to be significant predictors of early or late sports resumption.

Ethics Committee: our study was approved by independent ethics committee at Brest University.

\section{Results}

Study population: we included 120 patients, 108 men and 12 women, aged 13 to 61 years (mean, $27.7 \pm 9.6$ years). Among them, $35(29.2 \%)$ competed on a national or international level and $84(70 \%)$ at a sub-national level; the remaining patient was a recreational athlete. Of the 120 patients, 55 (45.8\%) had a previous history of one or more muscle injuries, including 42 with hamstring injuries more than 12 months earlier, 10 with quadriceps injuries, 4 with adductor injuries, and 2 with triceps surae injuries (1 patient sustained three muscle injuries, involving the hamstring, quadriceps, and adductors, respectively; and another patient sustained two muscle injuries, involving the hamstring and quadriceps, respectively). The distribution of injuries by sport is shown in Table 1. Of the 120 injuries, 48 (40\%) occurred during training and $72(60 \%)$ during competitions. Mean time from activity initiation to injury was $38 \pm 23$ minutes. The fitness level just before the injury, as assessed on a 0-10 scale by the patients, was high $(6.8 \pm 1.3)$. Of the 120 injuries, $74(61.7 \%)$ occurred during running, 42 (35\%) during sports-specific movements (e.g., shooting or tackling), and 4 (3.3\%) while jumping. Pain before the injury (pre-injury symptom) was reported in $32(26.7 \%)$ cases. All patients adhered fully to the rehabilitation programme.

Time to recovery: the time from injury to recovery of the pre-injury level of sporting activity was 40 days or less in $65(54.2 \%)$ patients and more than 40 days in

Table 1. Distribution of the 120 athletes with hamstring injuries by sport.

\begin{tabular}{ll}
\hline Sport & Number of injuries (\%) \\
\hline Soccer & $68(56.6 \%)$ \\
Rugby & $15(12.5 \%)$ \\
Running & $14(11.7 \%)$ \\
Handball & $8(6.7 \%)$ \\
Basketball & $3(2.5 \%)$ \\
Other & $12(10 \%)$ \\
\hline
\end{tabular}


$55(45.8 \%)$ patients. In the early recovery group, patients were able to resume jogging by day $13.7 \pm 5.8$ and the pre-injury level of sporting activity (including competitions) by day $24.0 \pm 7.9$. Corresponding times in the late recovery group were $34.0 \pm 12$ and more than 40 days, respectively.

Clinical criteria at baseline: the findings from the baseline evaluation are reported in Table 2. Mean VAS pain score was $5.7 \pm 1.9$ (range, 1-10). Pain scores greater than 6 were more common in the laterecovery group. Pain during everyday activities was present for $2.4 \pm 2.0$ days, and pain for more than 3 days was associated with late recovery. Other criteria associated with late recovery were a popping sound during the injury $(n=30,25 \%)$ and bruising $(n=18,15 \%)$. Pain during passive straightening of the limb ( $n=102,85 \%)$ was not associated with late recovery. The mean motion-range difference between the injured and the uninjured leg during passive straightening of the limb was $15.3^{\circ} \pm 14.3^{\circ}$ and a greater than $15^{\circ}$ difference $(n=54,45 \%)$ was associated with late recovery. Neither pain upon isometric contraction $(n=8,66 \%)$ nor tenderness to palpation $(n=89,74 \%)$ was associated with late recovery. The distance between the highest site of pain to palpation and the palpated ischial tuberosity was measured in 86 patients and was not associated with time to recovery (Tab. 3). All those clinical signs are recorded by physical examination within 5 days of onset injury.

By univariate analysis, five clinical criteria at baseline were associated with late recovery: VAS score for worst pain intensity greater than 6, popping sound injury, pain during everyday activities for more than 3 days, bruising, and greater than $15^{\circ}$ motion-range limitation compared to the uninjured side. By multivariate analysis, two criteria were significantly associated with late recovery, namely, initial VAS pain score greater than 6 and pain during everyday activities for more than 3 days. These two criteria were $53 \%$ sensitive and $95 \%$ specific for late recovery.
Table 3. Distance (in $\mathrm{cm}$ ) between the highest tender site upon palpation and the palpated ischial tuberosity, in five $10-\mathrm{cm}$ categories. Of the 89 patients with tenderness to palpation, 86 were evaluated for this parameter.

\begin{tabular}{lll}
\hline Distance $(\mathrm{cm})$ & Early recovery & Late recovery \\
\hline 0 to $<10 \mathrm{~cm}$ & 7 & 4 \\
10 to $<20 \mathrm{~cm}$ & 12 & 15 \\
20 to $<30 \mathrm{~cm}$ & 17 & 12 \\
30 to $<40 \mathrm{~cm}$ & 9 & 6 \\
$>40 \mathrm{~cm}$ & 2 & 2 \\
Total (86 patients) & 47 & 39 \\
\hline
\end{tabular}

\section{Discussion}

Hamstring muscle injuries are among the most common injuries occurring in sports ${ }^{14}$ and the best means of preventing them is unclear ${ }^{15-17}$. Sporting activities that require repeated bouts of sprinting often cause hamstring injuries ${ }^{18}$. In our study, $69 \%$ of the patients played soccer or rugby, both of which require sprinting. All our patients had had to stop their sporting activity immediately due to the sudden-onset of severe pain in the posterior thigh, and all exhibited unambiguous signs of hamstring injury by physical examination. In keeping with previous data, most of our patients were young men, who had warmed up properly, and considered themselves to be in good shape; and a larger proportion of our patients sustained the hamstring injury during competition than during training 2,4,6,19.

Imaging studies may not be necessary in all patients to establish the diagnosis and assess the prognosis of hamstring injuries. According to a 2005 literature review $^{20}, \mathrm{MRI}$ and ultrasonography are somewhat helpful (and perhaps unavoidable) in elite athletes (29.2\% of our patients) but should carry less weight than the clinical assessment. In keeping with a previous study ${ }^{4,5}$, the present multicenter study of patients

Table 2. Clinical severity criteria by physical examination in the groups with early and late recovery of the pre-injury level of performance ( $\leq 40$ days $v s>40$ days, respectively). The data are the numbers of patients (\%).

\begin{tabular}{lll}
\hline & Early recovery $n=65$ & Late recovery $n=55$ \\
\hline Worst VAS pain score $>6(n=69)$ & $23(19.2 \%)$ & $46(38.3 \%)^{*}$ \\
Pain of any severity for $>3$ days during every day activities $(n=48)$ & $16(13.3 \%)$ & $32(26.7 \%)^{*}$ \\
Popping sound at injury $(n=30)$ & $8(6.7 \%)$ & $22(18.3 \%)^{*}$ \\
Bruising $(n=18)$ & $5(4.2 \%)$ & $13(10.8 \%)^{*}$ \\
Tenderness upon palpation $(n=89)$ & $48(40 \%)$ & $41(34.2 \%)$ \\
Pain upon isometric contraction $(n=80)$ & $45(37.5 \%)$ & $35(29.2 \%)$ \\
Pain upon passive limb straightening $(n=102)$ & $52(43.3 \%)$ & $50(41.7 \%)$ \\
Limitation $>15^{\circ}$ to uninjured limb $(n=54)$ & $19(15.8 \%)$ & $35(29.2 \%)^{*}$
\end{tabular}

VAS, visual analog scale

${ }^{*} \mathrm{P}<0.05$ between the two groups 
with injuries to a single muscle group shows that the baseline clinical examination can provide valuable information about hamstring strain severity. We identified five criteria that are easy to assess during the patient interview and physical examination and that were associated with a longer time to recovery of full function (VAS pain score greater than 6, popping sound injury, pain during everyday activities for more than 3 days, bruising, and greater than $15^{\circ}$ motionrange limitation compared to the uninjured side). By multivariate analysis, two of these criteria were independently associated with a longer time to recovery (initial VAS pain score greater than 6 and pain during everyday activities for more than 3 days). In studies of Australian Rules football players, greater pain severity was associated with a longer absence from competition ${ }^{2,9}$, and taking more than 1 day to walk pain-free was associated with more than 3 weeks' absence from competition ${ }^{10}$. Similarly, we found that a VAS score for the worst injury-related pain greater than 6 and more than 3 days of pain during everyday activities (e.g., walking, dressing, or getting in and out of a car), were significantly and independently associated with needing more than 40 days to recover pre-injury function.

The only physical finding associated with time to recovery was greater than $15^{\circ}$ motion-range limitation compared to the uninjured side. This criterion was significant in the univariate analysis but not in the multivariate analysis. Several other tests are widely used to assess hamstring injuries ${ }^{21}$. In 165 elite track-and-field athletes, restricted active range of motion at the knee predicted time to recovery ${ }^{11}$. However, in this study, minor and major injuries were defined as restriction of less than $20^{\circ}$ and more than $30^{\circ}$, respectively; in addition, only 6 patients had major injuries and among them only 1 required more than 6 weeks to recover pre-injury function. Thus, the severity of the injuries was clearly greater in our patient population.

Our results suggest that the best time to assess athletes with suspected hamstring injuries may be the third or fourth day after the injury. The RICE proto$\mathrm{col}^{22}$ for minimizing hematoma formation immediately after muscle injuries is typically used for 2 days by athletes with suspected muscle injuries ${ }^{4}$. On the following day, the athletes usually have a clear recollection of the intensity of the initial pain, worst pain (a criterion used in our study), and whether a popping sound was heard at the time of injury. Bruising usually takes 2 to 5 days to develop. Finally, on the third or fourth day, difficulties with everyday activities such as walking and dressing can be easily assessed.

The anatomic structures damaged during initial and recurrent hamstring injuries ${ }^{18}$ have a strong influence on the time to recovery of the pre-injury level of performance. Injuries involving an intramuscular tendon or a fascia and adjacent muscle fibers typically require a shorter recovery period than those involving a proximal free tendon ${ }^{13,14,23}$. In our study, we used the distance between the highest tender site on the thigh and the palpated ischial tuberosity to estimate the anatomic site of injury ${ }^{14}$. This parameter was not associated with time to recovery and there was no predominant site of injury. The highest tender site may fail to accurately reflect the site of greatest hamstring damage.

Accurately predicting time to recovery is crucial in all injuries, particularly in athletes. The goal of the clinical examination and rehabilitation program is to return the athlete to the pre-injury level of performance quickly but with the smallest possible risk of recurrence. The baseline clinical findings may be less useful for predicting the risk of recurrence than for predicting the time to recovery. In a previous study, the 1-year recurrence rate was not significantly different between the groups with early and late recovery ( $\leq 40$ days or $>40$ days, respectively $)^{4,5}$. In elite track-and-field athletes, the risk of recurrent hamstring injury was greater with low-grade than with high-grade muscle lesions ${ }^{11,24,25}$. Injured athletes and their teams need to know how long the recovery process will take. Although an algorithm has been developed ${ }^{26}$, there are no universally accepted guidelines or criteria for the safe return to sports following muscle strain injuries ${ }^{20}$. Moreover, some athletes may return to their sporting activity before achieving a full recovery ${ }^{12}$.

Our study has several limitations ${ }^{27}$. First, neither ultrasonography nor MRI was used routinely. The size of the muscle lesion determined on imaging studies may be of crucial importance in determining the duration of the rest and rehabilitation program ${ }^{3}$. However, the few available studies of acute hamstring injuries produced somewhat conflicting results regarding the role for lesion size, healing time ${ }^{14}$ and recurrence risk $^{10}$. In 58 professional Australian football players, MRI was not required to estimate the duration of rehabilitation after an acute minor or moderate hamstring injury ${ }^{12}$. Ultrasonography is being increasingly used by specialists, most notably for the assessment of musculoskeletal disorders. When appropriately used, diagnostic ultrasonography serves as an extension of the physical examination. In a previous study, we showed that combining a clinical examination with an ultrasonographic assessment was an excellent method for predicting the time to recovery ${ }^{5}$. The results of the present study indicate that the clinical examination alone adequately predicts the time to recovery. Second, the time from hamstring injury to resumption of competitions may be influenced by factors other than injury severity. In our study, all the patients followed the same rehabilitation program but the physical therapists differed across patients. However, this well-recognized limitation to longitudinal studies of hamstring injuries is nearly unavoidable $e^{2,13}$. Third, of our 120 patients, only 39 were elite athletes and only 1 was a recreational athlete. Nevertheless, the presentation of acute hamstring injury is not likely to differ between elite and other athletes, and neither are there any substantial differences in therapeutic management, given the very low level of the scientific evidence available to support experimental treatments. 
Perspective: VAS pain score greater than 6 (worst injury related VAS pain score) and pain during everyday activities for more than 3 days are significantly associated with time to recovery $>40$ days. This identification of these items which may predict outcome is a first step for further evaluation combining clinical and imaging data. The potential impact of the present findings is the definition of a core set predicting the time to recovery using only clinical sign. The evaluation of these criteria in combination with $\mathrm{MRI}^{7,9}$ should be done.

\section{Conclusion}

In conclusion, in this study of 120 athletes with acute hamstring injuries, the baseline clinical examination provided valuable information for predicting the time to recovery of the pre-injury level of performance.

\section{Practical Implications:}

- Sporting activities are the leading cause of muscle injuries, among which hamstring strain is the most common.

- The initial clinical examination provides valuable information that can be used to predict the time to full recovery after acute hamstring injuries in athletes.

- VAS pain score greater than 6 and pain during everyday activities for more than 3 days are significantly associated with time to recovery $>40$ days, with $53 \%$ sensitivity and $95 \%$ specificity.

\section{References}

1. Ekstrand J, Hägglund M, Waldén M. Epidemiology of muscle injuries in professional football (soccer) Am J Sports Med. 2011;39:1226-1232.

2. Verrall GM, Slavotinek JP, Barnes PG, Fon GT. Diagnostic and prognostic value of clinical findings in 83 athletes with posterior thigh injury: comparison of clinical findings with magnetic resonance imaging documentation of hamstring muscle strain. Am J Sports Med. 2003;31:969-973.

3. Koulouris G, Connell D. Imaging of hamstring injuries: therapeutic implications. EurRadiol. 2006;16:1478-1487.

4. Guillodo Y, Saraux A. Treatment of muscle trauma in sportspeople (from injury on the field to resumption of the sport). Ann Phys Rehabil Med. 2009;52:246-255.

5. Guillodo Y, Boutier R, Saraux A. Value of sonography combined with clinical evaluation to determine the severity of skeletal muscle injuries in sportsmen. J Athl Train. 2011:46(5):527-532.

6. Orchard JW. Intrinsic and extrinsic risk factors for muscle strains in Australian football. Am J Sports Med. 2001;29:300303.

7. Silder A, Heiderscheit BC, Thelen DG,Enright T, Tuite MJ. MR observations of long-term musculotendon remodelling following a hamstring strain injury. Skeletal Radiol. 2008;37:11011109.

8. Mjølsnes R, ArnasonA, Østhagen T, Raastad T, Bahr R. A 10week randomized trial comparing eccentric vs concentric ham- string strength training in well-trained soccer players. Scand $\mathrm{J}$ Med Sports. 2004;14:311-317.

9. Verrall GM, Slavotinek JP, Barnes PG, Fon GT, Esterman A. Assessment of physical examination and magnetic resonance imaging findings of hamstring injury as predictors for recurrent injury. J Orthop Sports Phys Ther. 2006;36:215-224.

10. Warren P, Gabbe BJ, Schneider-Kolsky M, Bennell KL. Clinical predictors of time to return to competition and of recurrence following hamstring strain in elite Australian footballers. $\mathrm{Br} \mathrm{J}$ Sports Med. 2010;44:415-419.

11. Malliaropoulos N, Papacostas E, Kiritsi O, Papalada A, Gougoulias N, Maffuli N. Posterior thigh muscle injuries in elite track and field athletes. Am J Sports Med. 2010;38:1813-1819.

12. Schneider-Kolsky ME, Hoving JL, Warren P, Connell DA. A comparison between clinical assessment and magnetic resonance imaging of acute hamstring injuries. Am J Sports Med. 2006,34:1008-1015.

13. Askling C, Saartok T, Thorstensson A. Type of acute hamstring strain affects flexibility, strength, and time to return to pre-injury level. Br J Sports Med. 2006;40:40-44.

14. Askling $\mathrm{C}$, Tengvar M, Saartok $\mathrm{T}$, Thorstensson A. Acute firsttime hamstring strains during high-speed running: a longitudinal study including clinical and magnetic resonance imaging findings. Am J Sports Med. 2007;35:197-206.

15. Engebretsen AH, Myklebust G, Holme I, Engebretsen L, Bahr R. Intrinsic risk factors for hamstring injuries among male soccer players: prospective cohort study. Am J Sports Med. 2010;38:1147-1153.

16. Fousekis K, Tsepis E, Poulmedis P, Athanasopoulos S, Vagenas $\mathrm{G}$. Intrinsic risk factors of non-contact quadriceps and hamstring strains in soccer: a prospective study of 100 professional players. Br J Sports Med. 2010;45:709-714.

17. Goldman EF, Jones DE. Interventions for preventing hamstring injuries. Cochrane Database Syst Rev. 2010;(1): CD006782.

18. Heiderscheit BC, Sherry MA, Silder A, Chumanov ES, Thelen DG. Hamstring strain injuries: recommendations for diagnosis, rehabilitation and injury prevention. J Orthop Sports Phys Ther. 2010;40:67-81.

19. Woods C, Hawkins RD, Maltby S, Hulse M, Thomas A, Hodson A. Football Association Medical Research Programme. The Football Association Medical Research Programme: an audit of injuries in professional football-analysis of hamstring injuries. Br J Sports Med. 2004;38:36-41.

20. Orchard J, Best TM, Verrall GM. Return to play following muscle strains. Clin J Sport Med. 2005;15:436-441.

21. Zeren B, Oztekin HH. A new self-diagnostic test for biceps femoris muscle strains. Clin J Sports Med. 2006;16:166-169.

22. Järvinen $T A$, Järvinen $T L$, Kääriänen $M$, et al. Muscle injuries: optimising recovery. Best Pract Res Clin Rheumatol. 2007;21:317-331.

23. Askling C, Tengvar M, Saartok T, Thorstensson A. Proximal hamstring strains of stretching type in different sports: injury situations, clinical and magnetic imaging characteristics, and return to sport. Am J Sports Med. 2008;36:1799-1804.

24. Malliaropoulos N, Isinkaye T, Tsitas K, Maffuli N. Reinjury after acute posterior thigh muscle injuries in elite track and field athletes. Am J Sports Med. 2011;39:304-310.

25. Malliaropoulos NG. Non contact hamstring injuries in sports. Muscles Ligaments Tendons J. 2013;2(4):309-311.

26. Mendiguchia J, Brughelli M. A return-to-sport algorithm for acute hamstring injuries. Phys Ther Sport. 2011;12:2-14.

27. Padulo J, Oliva F, Frizziero A, Maffulli N. Muscles, Ligaments and Tendons Journal. Basic principles and recommendations in clinical and field science research. MLTJ. 2013;4:250-252. 